\title{
La road movie como modelo transnacional y su presencia en el cine español: marco metodológico y principales aportaciones
}

\author{
Santiago García Ochoa \\ Universidad de Santiago de Compostela \\ santiagogarciaochoa@gmail.com
}

RESUMEN: El presente artículo defiende que los estudios transnacionales constituyen el marco metodológico idóneo para el estudio de la espectacular difusión de la road movie, un género nacido en EE. UU. durante la década de los 60, al menos a través de tres vías: la contraposición entre lo nacional y lo transnacional, lo transnacional entendido como fenómeno regional y el protagonismo de las migraciones en los cines postcoloniales. Finalmente se aborda el desarrollo de la road movie en España y se repasan los principales trabajos académicos sobre el tema.

PALABRAS CLAVE: Road Movie, Estudios transnacionales, Cine español, Hollywood.

\section{The Road Movie as a Transnational Model and its Presence in Spanish Cinema: Methodological Framework and Main Contributions}

ABSTRACT: The present article defends that transnational studies are the most appropriate methodological framework to study the presence of the road movie (an American genre born during the sixties) worldwide through at least three ways: the binary of national/transnational, the transnational as a regional phenomenon and the diasporic, exilic and postcolonial cinemas. Finally, the author tackles the Spanish road movie and the main academic literature on this topic.

KEYWORDS: Road Movie, Transnational Studies, Spanish Cinema, Hollywood.

Recibido: 16 de febrero de 2016 / Aceptado: 8 de junio de 2016.

\section{Un género impuro}

La mayor parte de los autores coinciden en que la road movie surge y se desarrolla como un modelo norteamericano íntimamente ligado a algunas de las principales constantes históricas y culturales de EE. UU.: la teoría de la frontera, el highway system, la preponderancia del automóvil, el western, Kerouac y la Beat Generation, etc ${ }^{1}$.

En cualquier caso, habría que distinguir entre los que sitúan su nacimiento a finales de los sesenta (dentro del periodo conocido como New Hollywood)2 , tendencia mayoritaria a partir de Corrigan (1991) a la que nos sumamos, y los que prescinden de una acotación cronológica clara, como Steven Cohan e Ina Rae Hark, que consideran la road movie como un género más del cine norteamericano de los años treinta (al igual que el western o el musical)3: «The impact of Easy Rider is undeniable and important to any understanding of the genre, but it has also obscured the road movie's own history» (Cohan \& Hark, 1997: 4).

GARCÍA OCHOA, Santiago: «La road movie como modelo transnacional y su presencia en el cine español: marco metodológico y principales aportaciones», Boletín de Arte, n. ${ }^{\circ}$ 37, Departamento de Historia del Arte, Universidad de Málaga, 2016, pp. 79-88, ISSN: 0211-8483. 
New Hollywood (1967-1975) supuso la materialización de grandes cambios en la organización industrial y económica de Hollywood, en gran medida por la competencia del medio televisivo, pero también la entrada de la modernidad cinematográfica, debida a la influencia de los «nuevos cines» europeos ${ }^{4}$. Por fin, el director dejaba de ser considerado un mero engranaje dentro del studio system para pasar a convertirse en el verdadero protagonista del proceso de producción de una película, en «el autor de la obra». En otras palabras: por primera vez en Hollywood la etiqueta de cine de autor se imponía a la de cine de género.

El concepto de género cinematográfico se ve también inevitablemente alterado desde finales de los sesenta por la diversificación del público, imposible de reducir a un modelo unitario. De todos los grupos sociales, el que cobra ahora un mayor protagonismo es el de los jóvenes. El incremento de los recursos económicos tras la II Guerra Mundial y la larga etapa de formación condujo a que los adolescentes (y los «jóvenes adultos» para el caso de los universitarios) fuesen creando unas señas de identidad propias basadas en la rebeldía frente a los valores defendidos por los adultos y por el propio sistema vigente, al que identifican con sus padres: las señas conocidas como contracultura. Su mirada se encontraba ya hipertrofiada por la constante repetición en la pantalla, tanto cinematográfica como televisiva, de las mismas convenciones genéricas. Corrigan (1991: 141-143) bautizó este fenómeno como "Generic Hysteria», tomando el sentido del término «histeria» de Derrida

La road movie nace pues como un fenómeno genérico impuro, de naturaleza intertextual, un «intergénero» según Sánchez Noriega (2002)5; no en vano las «películas de carretera» siempre se pueden enmarcar claramente dentro de, al menos, uno de los géneros clásicos: drama, comedia, policíaco, etc.

\section{Beyond USA: La road movie en el marco de los estudios transnacionales}

Los especialistas apenas se interesaron por el fenómeno de la importación del género road movie hasta la última década del siglo XX, cuando se produjo una espectacular proliferación de este tipo de películas a nivel mundial.

Dos son los factores esenciales que otorgan a la road movie un carácter universal según Michel Euvrard: el prime- ro, el reflejo de la estandarización de los modos de vida en los países industrializados según el modelo norteamericano: economía dominada por la industria del automóvil y sus consecuencias, autopistas, estaciones de servicio, etc.; y segundo, la capacidad de adaptación de este género, estructura y síntoma al mismo tiempo, que puede ser revisado sin perder sus raíces, que jamás sacrifica su tema central, esto es, el viaje, la búsqueda o el vagabundeo, aunque en ocasiones se desvanezcan algunas de sus características (Euvrard, 1988: 34).

Walter Moser considera que si bien la road movie conoció una primera formulación esencialmente estadounidense, a partir de los años setenta, en el contexto de la mundialización, se difundió a todos los países y continentes, favoreciendo de forma especial el cruce de culturas, la interculturalidad: "[...] la déprise, le mouvement vers l'inconnu, la contingence radicale vécue par le protagoniste, peut se traduire en une disposition particulièrement ouverte pour la rencontre avec l'Autre culturel, avec d'autres cultures» (Moser, 2008: 27).

No en vano, con cierta frecuencia se ha recurrido a los estudios transnacionales para analizar la presencia de la road movie dentro de las diferentes cinematografías nacionales. El concepto transnacional, originalmente aplicado en economía a aquellas empresas que operan en varios países, conoció un interesante desarrollo dentro del ámbito académico norteamericano desde principios de los noventa, aplicado al estudio de los fenómenos migratorios: de las relaciones económicas, políticas y sociales que los inmigrantes mantenían con sus lugares de acogida y origen, concediéndole un importante papel al territorio, de lo local a lo nacional, en la configuración de las comunidades y la producción de significados culturales. La teoría transnacional también fue aplicada al estudio de la producción cultural en el mundo globalizado, con el cine como campo de estudio privilegiado, incluyendo al ámbito del hispanismo. Así, los editores del número monográfico del Hispanic Research Journal «The Transnational in Iberian and Latin American Cinemas», Ilaman la atención sobre cómo los nuevos directores ponen en cuestión la tradicional definición de cine nacional a través del entrecruzamiento de las identidades globales y locales (Perriam, Santaolalla \& Evans, 2007: 4).

Higbee y Lim distinguen tres posibles vías de trabajo con las películas desde la perspectiva de los estudios trans- 
nacionales: la primera está centrada en el binomio nacional/ transnacional, en las dificultades para enmarcar las relaciones culturales y económicas dentro de los límites tradicionales de lo nacional; la segunda comprende el análisis de lo transnacional como un fenómeno regional, preguntándose si es posible hablar de un cine chino supranacional o de un cine paneuropeo; y la última propuesta se centra en el estudio de los cines postcoloniales del exilio y la diáspora, que construyen una identidad nacional propia como alternativa a la cultura eurocéntrica dominante (Higbee \& Lim, 2010: 9).

La primera de las vías descritas, tradicionalmente enfocada a cuestiones relacionadas con la producción, la distribución y la exhibición, se puede aplicar al estudio de la adopción de un modelo genérico norteamericano por parte de otras cinematografías. Eyerman y Löfgren (1995) fueron de los primeros en preguntarse qué sucede cuando la road movie es trasplantada a un contexto social y cultural diferente del norteamericano.

Los autores de este pionero artículo dedican varias páginas a analizar el género road movie en EE. UU. y su evolución, para concluir que, a pesar de la aparición, desde principios de los noventa, de títulos suecos como Ha ett underbart liv (Have a Wonderful Life, Ulf Malmros, 1991) o Drömmen om Rita (Dreaming of Rita, John Lindström, 1992), que se acercan al modelo norteamericano, las diferencias ideológicas sobre la movilidad social en EE.UU., como el predominio de lo individual, la aceptación de las diferencias de clase, la promoción a través de la iniciativa privada, y en Suecia, predominio de lo colectivo, negación de las clases por el desarrollo del estado de bienestar, ascenso social vinculado al sector público, son profundas e insalvables (Eyerman \& Löfgren, 1995: 76-77). No obstante, la crisis del estado de bienestar en los noventa podría favorecer que la road movie, con su mensaje de futuro y su fascinación por lo desconocido, encuentre un nuevo público, tanto en Suecia como en el resto de Europa: «The road movie is a genre tailored for tales and times of crisis -for downward as well as upward mobility- whether they be individual or collective» (Eyerman \& Löfgren, 1995: 77) ${ }^{6}$.

Susan Picken entiende que las peculiares condiciones geográficas de Gran Bretaña («Enclosed within this coastal barricade, Britain is a small island dominated by an abundance of water») justifican la inexistencia de una highway culture como la que alimentó el género en EE. UU. El paisaje británico es funcional y pragmático, sin nada comparable a los grandes espacios abiertos norteamericanos, como el desierto de Arizona: «No inspiration here for an On the road or Easy rider, we prefer On the buses (Harry Booth, 1971)» (Picken, 1999: 222-223).

Según Neil Archer la road movie francesa de los últimos años ha contribuido a poner en crisis los conceptos de nación y cine nacional, cada vez más paradójicos en un mundo globalizado: el deseo de viajar es internacional y las películas podrían estar ambientadas en cualquier país moderno (2013: 172). Las transformaciones espaciales provocadas en Europa por la generalización del uso del automóvil durante la segunda mitad del siglo XX, capítulo esencial de la americanización postbélica, son las principales responsables de esta homogeneización espacial. Para Dupuy la incorporación del automóvil ha provocado transformaciones espaciales cuantitativas y cualitativas irreversibles. La característica más llamativa de lo que él denomina «sistema del automóvil» es la estandarización: el coche ha hecho desaparecer en gran medida la diversidad territorial más allá de las fronteras nacionales (Dupuy, 1995: 185 y 188).

La adaptación del género road movie a unas determinadas coordenadas nacionales puede ser más o menos profunda dependiendo de la evolución sociopolítica y cultural del país, pero también de su nivel de implicación en el sistema mundial de la globalización; evidentemente los rasgos característicos del género road movie han cuajado más y mejor en las cinematografías europeas que en las asiáticas o en las africanas.

Desde la segunda vía se puede plantear la existencia de una "road movie paneuropea" como una alternativa al modelo norteamericano. De hecho, diversos especialistas se han decantado claramente por este enfoque. David Laderman enumera los siguientes rasgos distintivos: la presencia de los personajes en la carretera se debe más a una necesidad imperiosa, de buscar trabajo o un hogar, que a su libre elección; la sustitución del protagonismo masculino y de la pareja de delincuentes por el viaje en grupo: y la práctica inexistencia de un universo simbólico asociado al coche y al acto de conducir, ya que en Europa el sentido que adquiere el viaje anula al medio de transporte empleado (Laderman, 2002: 248).

Mazierska \& Rascaroli (2006: 4) admiten que por su extrema riqueza y diversidad las películas de carretera eu- 
ropeas no constituyen un género coherente, como en el ámbito norteamericano, pero al establecer una serie de diferencias entre ambos casos contribuyen a definir un modelo de «road movie paneuropea»: los espacios abiertos de Norteamérica con su sistema de autopistas y su sentido de la libertad contrasta claramente con una Europa formada por un mosaico de naciones, culturas e idiomas separados por fronteras geográficas y políticas; los medios de transporte privilegiados en el cine norteamericano son el coche particular y la moto, mientras que en el europeo predomina el transporte público, esto es, autobuses y trenes, el autostop o el viaje a pie; los personajes de las road movies norteamericanas suelen ser rebeldes en busca de libertad o a la fuga, mientras en las europeas se trata de ciudadanos corrientes que se desplazan por un motivo concreto, como vacaciones, trabajo, etc. (Mazierska \& Rascaroli, 2006: 5).

Un importante legado cultural, la novela picaresca (a partir del Lazarillo de Tormes), El Quijote y la Bildungsroman («novela de formación»), avalan la tesis de la alternativa europea. Además de que, como hemos visto, varios títulos de los «nuevos cines» que, evidentemente, recogen esta tradición, prefiguran el modelo norteamericano ${ }^{7}$.

En cualquier caso, plantear la existencia de una «road movie paneuropea» como alternativa al modelo norteamericano choca frontalmente con el concepto de cine nacional. Si bien es cierto que los países europeos han experimentado los mismos cambios sociales, mentales y económicos en la segunda mitad del siglo $X X$, pues Europa puede considerarse como una realidad económica e incluso política, también lo es en la misma medida que esta experiencia común no ha hecho desaparecer las diferencias culturales, ni la coyuntura específica de cada país. Similitudes y diferencias se filtran y se yuxtaponen pues en las películas europeas (Sorlin, 1996: 13-14).

Desde un punto de vista más restrictivo, regional, Andrew Nestingen habla de la Nordic road movie, que ha aprovechado la fijación en la movilidad y los encuentros propia del modelo norteamericano para unificar fronteras e identidades. Las diferencias entre las películas norteamericanas y las escandinavas, especialmente en lo que se refiere al paisaje, espacios abiertos frente a espacios claustrofóbicos, obligan a replantearse la relación entre cine nacional y géneros cinematográficos: "The images of the road movie and Nordic national landscapes fit together in an intriguing and thought-provoking connection» (Nestingen, 2005: 280).
La tercera de las vías propuestas relaciona los cines periféricos con la esencia misma de la road movie: el exilio, la emigración, el nomadismo o la diáspora, tan presentes en las cinematografías asiáticas, africanas o latinoamericanas de las últimas décadas, en las que el recurso a lo transnacional, a un modelo/género internacionalmente aceptado, ha contribuido a llamar la atención de crítica y público sobre unas culturas más o menos desconocidas. Ute Fendler (2008) considera que las road movies producidas en el África francófona a partir de los años noventa se inscriben en unas tradiciones propias sobre los viajes iniciáticos, aunque también demuestran la diversidad étnica y cultural africana, ilustrando los cambios de unas sociedades en vías de desarrollo: mientras la emigración Sur-Norte se relaciona con un futuro mejor, la Norte-Sur aparece asociada a la búsqueda de las raíces perdidas.

Pero los fenómenos asociados a desplazamientos masivos y constantes de población, como exilio, emigración o diáspora, también han afectado a la historia reciente de Europa, cuyas transformaciones políticas, sociales y económicas incluyen la caída del comunismo, la desintegración de la Unión Soviética y de la Europa del Este, el postfordismo, la globalización, el ensanchamiento de la Unión Europea, la reconfiguración de fronteras y el problema de los refugiados (conflicto de los Balcanes), el incremento de la inmigración procedente de países del Tercer Mundo, etc. (Mazierska \& Rascaroli, 2006: 1-2).

En concreto, Elizabeth Mittman plantea interesantes similitudes entre cómo abordan algunas películas de principios de los noventa el viaje por la Alemania reunificada y la poética de la road movie. Comedias como Go Trabi Go (Peter Timm, 1991) y Wir können auch anders (No More Mr. Nice Guy, Detlev Buck, 1993) comparten una serie de elementos comunes con los films norteamericanos: el viaje, los conflictos que surgen de la relación con los extraños, la profundización de los lazos entre los viajeros o la iconografía del automóvil. En cualquier caso, películas como estas proporcionan un campo de referencias muy amplio para la interpretación de un proceso tan complejo a todos los niveles (político, social, psicológico) como el de la reunificación: «As East and West Germans travel into the other half of Germany -each effectively moving from home into a foreign countrythey perform an act of rejection, or of leaving something behind» (Mittman, 2003: 329). 


\section{El cine español on the road}

En el caso del cine español, la configuración de la carretera como un espacio de búsqueda individual y colectiva se produce tras la muerte de Franco, por lo que las primeras road movies aparecen durante el periodo de la transición: El puente (Juan Antonio Bardem, 1977), Vámonos, Bárbara (Cecilia Bartolomé, 1978), A contratiempo (Óscar Ladoire, 1981) y Corridas de alegría (Gonzalo García Pelayo, 1981).

El puente y Vámonos, Bárbara recurren a los códigos de dos subgéneros de explotación que triunfaban en los setenta, la comedia sexy landista y la película erótica (poco le faltó al film de Bartolomé para ser clasificado "S») respectivamente, para descargar sobre el espectador una pesada carga ideológica de signo izquierdista (sobre la legalización de los sindicatos en el primer caso y la liberalización de la mujer en el segundo), en un momento en el que, aunque la transición democrática estaba ya en marcha, la realidad legal y social de España seguía siendo en gran medida franquista. El puente y Vámonos, Bárbara incorporan además códigos muy reconocibles de la road movie que potencian notablemente su intencionalidad reivindicativa: tanto la estructura -el tránsito constante y las paradas significativas-, como la ideología -la denuncia social de un sistema que deja fuera a determinadas minorías- de la primeras road movies producidas en el seno de New Hollywood cuadran a la perfección con los intereses éticos y estéticos de Juan Antonio Bardem y Cecilia Bartolomé. En ambos títulos la carretera adquiere pues un claro valor metafórico: es el camino que deben recorrer los españoles hasta la plena instauración de la democracia.

Tan sólo unos meses antes del triunfo socialista, se estrenan las otras dos road movies emblemáticas de la transición: A contratiempo, que plantea una nueva alianza entre la comedia y la road movie, en este caso, como vía de escape del escepticismo y el desencanto, el «individualismo pasota", de la generación de la movida madrileña; y Corridas de alegría, sin duda, la road movie más contracultural del cine español, el auténtico Easy Rider hispano ${ }^{9}$ en el que reaparecen la liberación sexual muy explícita: la película fue calificada "S», las drogas, e incluso el rock, aunque en su vertiente autóctona o «rock andaluz».

En cualquier caso, la proliferación de la poética de la road movie en el cine español no se produce hasta los años noventa, en plena globalización, coincidiendo con la revitalización de los géneros como fuente «noble» y su uso en clave postmoderna por los cineastas, con títulos tan diversos como Suspiros de España (y Portugal) (José Luis García Sánchez, 1994) y Airbag (Juanma Bajo Ulloa, 1997), que renuevan la relación de la road movie con la comedia; Dispara (Carlos Saura, 1993), Hola, ¿estás sola? (Iciar Bollaín, 1995) y Fugitivas (Miguel Hermoso, 2000), que convierten a la mujer en protagonista siguiendo la estela de Thelma and Louise (Thelma y Louise, Ridley Scott, 1991); o Carreteras secundarias (Emilio Martínez Lázaro, 1997) y Los años bárbaros (Fernando Colomo, 1998), que ponen el género al servicio del revisionismo histórico. En definitiva, la road movie aparece como un género compatible con los intereses personales de los cineastas, al que recurren con cierta frecuencia para articular sus hibridaciones, que tiene incluso cabida en un universo autoral tan cerrado como el de Antonio Eceiza (Felicidades, Tovarich, 1995).

En las películas del nuevo milenio se observa la presencia cada vez más recurrente de los nuevos marginados, como inmigrantes, ancianos, enfermos, a imitación del cine norteamericano ${ }^{10}$ : un niño negro huérfano en Nos hacemos falta -Tilt- (Juanjo Giménez Peña, 2002), un inmigrante marroquí en El rayo (Fran Araujo y Ernesto de Nova, 2013), una anciana cercana a los 80 y la hondureña que la cuida en Mami Blue (Miguel Ángel Calvo Buttini, 2010) o un paralítico con una enfermedad degenerativa y su cuidadora rumana en Anochece en la India (Chema Rodríguez, 2013).

Dos son las principales líneas de desarrollo de la road movie en los últimos años: la "comedia gamberra" de carretera y el cine de autor on the road. La primera, comercial y postmoderna, tiene su origen en la combinación de la «comedia gamberra» norteamericana con la road movie, y se alimenta de la estela dejada por Airbag ${ }^{11}$; entre sus títulos destacan: Slam (Miguel Martí, 2003), El síndrome de Svensson (Kepa Sojo, 2006) o Mami Blue. La segunda, basada en la experimentación formal y narrativa de sus creadores, ha dado lugar a películas como Dies d'agost (Días de agosto, Marc Recha, 2006), El muerto y ser feliz (Javier Rebollo, 2012) o El rayo.

Unos cuantos autores, casi siempre desde el ámbito académico del hispanismo, han aplicado el enfoque transnacional al estudio de la road movie en el cine español, tanto a través del análisis de películas concretas como en otro tipo 
de estudios, más ambiciosos, y todavía más escasos, que abarcan el fenómeno de una forma global.

Induráin define Airbag como un híbrido entre la road movie norteamericana, con sus clubs de carretera, casinos y paisaje versión española, todo ello enmarcado por un espectáculo de acción típicamente hollywoodiense, sin olvidar el viaje iniciático que realiza Juantxo (Karra Elejalde) acompañado de sus dos amigos, protagonismo masculino (Induráin, 2005: 117-118); y la comedia, el humor típicamente hispano: crítica de la iglesia católica, de la Guardia Civil y de la aristocracia, heredada de Berlanga y el primer Almodóvar, sin equivalente, lógicamente, en el modelo norteamericano (Induráin, 2005: 114-115). El artículo evidencia la dificultad de fijar un modelo más o menos rígido de híbrido, es decir de «road movie española», ya que si bien los elementos alóctonos, esto es, protagonismo del viaje por carretera, importancia de los encuentros, estructura episódica, etc., pueden resultar más o menos fáciles de evaluar, como hace Induráin, los autóctonos pueden variar notablemente de un film a otro: desde los puramente literarios, a partir de la novela picaresca, a los procedentes de estilos, géneros o autores concretos; desde los intentos de «nacionalizar» el género a las películas que buscan el éxito de taquilla a través de un modelo hollywoodiense claramente reconocible para el espectador. Más recientemente, Induráin (2016) ha vuelto sobre el fenómeno de la transnacionalidad de la road movie para determinar que la hibridación del modelo norteamericano con las constantes propias del cine español se desarrolla en plena consonancia con las dos tendencias predominantes a partir de los años noventa: la postmoderna, ejemplificada nuevamente con Airbag, y la realista, con Fugitivas.

Como modelo internacional, el género road movie resulta pues fácilmente definible, pero el establecimiento de modelos cinematográficos nacionales, que evidencien temas recurrentes y señas de identidad culturales, es complicado y también muy controvertido: solo hay que recordar, para el caso español, las simplificaciones reduccionistas en las que ha incurrido la literatura foránea, generalmente la anglosajona. Santos Zunzunegui es uno de los escasos historiadores que han intentado definir un modelo de cine nacional español con una base coherente, consciente de las contradicciones que se pueden llegar a generar ${ }^{12}$, pero convencido de que el principal objetivo de la historiografía del cine español debe ser:
[...] sacar a la luz la manera en que nuestro cine prolonga, desarrolla y reescribe (a la altura de cada momento histórico) toda una serie de elementos que forman una especie de «hilo rojo» que atraviesa las distintas prácticas culturales que se han sucedido en el impreciso marco de eso que para entendernos denominaré (entrecomillándolo cuidadosamente) «Cultura española» (Zunzunegui, 2011: 9).

Desde 2002, este historiador defiende la existencia de cuatro grupos de autores que configuran las «vetas» del cine español: el primero, el de los que huyen del realismo primario por la vía de la exageración (Marco Ferreri, Luis García Berlanga, Fernando Fernán Gómez), corriente que parte del Arcipreste de Hita y pasa por Quevedo, Goya o Valle Inclán; el segundo, los que, con Edgar Neville a la cabeza, recuperan ciertas prácticas de la cultura popular desde una perspectiva integradora; el tercero, los que, en contadas ocasiones, logran desbordar el realismo por la vía del mito, como Manuel Gutiérrez Aragón o Julio Medem; y el cuarto, los que mezclan armoniosamente la vanguardia con lo popular: Iván Zulueta o Joaquín Jordá (Zunzunegui, 2002) ${ }^{13}$.

Pérez (2008a) recupera las metáforas sobre la movilidad fraguadas en torno a la transición a la democracia y las aplica al análisis de Vámonos, Bárbara. Así, la carretera por la que transitan las protagonistas se convierte en la que conduce a las mujeres a la liberación sexual. El autor plantea una faceta inédita del cine de la transición que desarrollará más ampliamente en su libro (Pérez, 2011), el primero dedicado a estudiar el fenómeno de la road movie española, aunque no se ciñe en exclusiva a las películas, y estudia el road genre también en la literatura (demostrando la fértil relación entre ambos medios) ${ }^{14}$. Pérez parte de la metáfora de la rotonda, que da título a su obra: «[...] I propose to conceptualize the road genre in Spain as a cultural roundabout in which cultural traffic flows in different directions, allowing multiple points of entry and exit» (Pérez, 2011: 12). Lo cual, evidentemente, se traduce en el recurso a una metodología dinámica e interdisciplinar, y a una postura crítica frente a los estudios transnacionales:

The road genre flourishes both as a response to and as an expression of the sociopolitical and cultural changes in Spain at the turn of the century, and not merely as a result of the importation of American cultural codes (Pérez, 2011: 25). 
El autor defiende que el desarrollo del road genre en España, prescindiendo aquí de los ejemplos literarios, va unido a dos momentos trascendentales de crisis sociopolítica y cultural: la transición a la democracia, en su nacimiento, y los años noventa, con su triunfo definitivo. En el libro se analizan Vámonos, Bárbara y El puente como ejemplos que conceptualizan la metáfora de la carretera como vía indirecta, rotonda en vez de autopista, hacia la democracia; Los managers (Fernando Guillén Cuervo, 2006), como parodia de la road movie; Kasbah (Mariano Barroso, 2000), en la que se abandonan las carreteras españolas y aparece la interculturalidad; y, finalmente, Fugitivas, que ilustra el triunfo de la mujer al volante en un género tradicionalmente masculino.

Pohl (2007) aplica las teorías transnacionales al estudio de «un género internacional por antonomasia, la road movie» (54) que, aunque en el cine español se inaugura con El puente, no triunfa hasta la década de los 90:

En este contexto de fragmentación y globalización, la road movie se ofrece como un modo de afrontar cuestiones del momento [...] Vale, por ello, la pena considerar cómo [...] puede contribuir a la representación o transgresión de un paisaje nacional determinado (Pohl, 2007: 58).

Pohl considera que hay que tener en cuenta dos elementos para analizar una road movie española desde el enfoque transnacional: la sustitución de la banda sonora y el paisaje norteamericanos por la música folclórica y los paisajes desolados de la planicie manchega o andaluza (Pohl, 2007: 59-60). Señala también que, en ocasiones se transgrede el ámbito nacional, prolongando el viaje a otros países de Europa, África o América e introduciendo «[...] otras nacionalidades y etnias como elementos de una interacción transnacional» (Pohl, 2007: 60-61).

Por último, Lie (2011) pone en tela de juicio la acotación histórica y geográfica de la road movie (parte de Orgeron) al considerar que Stress es tres tres (1968), film muy representativo de la estética de los «nuevos cines», es ya una road movie; señala que la película de Saura es anterior a Easy Rider. La autora también incurre en una lectura sociológica aberrante al considerar que el protagonista, Fernando (Fernando Cebrián), representa el «prototipo del nuevo español progresista de los sesenta mediante su atributo más elocuente: el coche» (Lie, 2011: 35), cuando el personaje conduce un Dodge y pertenece a la clase dirigente (burguesía franquista).

\section{Conclusiones}

La road movie es un género íntimamente ligado a la cultura norteamericana que surge en el periodo conocido como New Hollywood (1967-1975), bajo el influjo de los «nuevos cines» europeos; es por ello que no debe ser considerado un género en la misma medida que lo son el western o el melodrama, sino como un «intergénero» (según Sánchez Noriega).

La road movie se exportó de forma inmediata a las cinematografías de todo el mundo, si bien su triunfo definitivo no se produce hasta la década de los 90. Frecuentemente se ha recurrido a los estudios transnacionales para estudiar este fenómeno. Aquí proponemos tres posibles vías de trabajo: la primera se centra en las fricciones entre lo nacional y lo transnacional; la segunda se interesa por la posibilidad de fijar modelos regionales de road movie, paneuropeo o nórdico; la tercera tiene en cuenta la importancia que adquieren el exilio y la diáspora en los cines periféricos, y en cómo el recurso a la road movie facilita la aceptación internacional de unas películas que construyen una identidad nacional ajena a la europea.

En España la aparición de la road movie se produce durante la transición a la democracia (1975-1982), con El puente, Vámonos, Bárbara, A contratiempo y Corridas de alegría; si bien su triunfo definitivo no tiene lugar hasta la segunda mitad de la década de los noventa, coincidiendo con la revitalización del cine de género, en títulos tan dispares como Carreteras secundarias, Airbag o Fugitivas. En la actualidad el fenómeno goza de muy buena salud, como lo demuestran Anochece en la India, El rayo, Vivir es fácil con los ojos cerrados (David Trueba, 2013) o Rey Gitano (Juanma Bajo Ulloa, 2015).

Muy pocos son los autores que hasta la fecha se han centrado en el estudio del desarrollo de la road movie dentro de la cinematografía española, tratándose casi siempre de artículos sobre películas concretas. El libro de Jorge Pérez Cultural Roundabouts: Spanish Film and Novel on the Road (2011) destaca por ser el primero dedicado a estudiar el fenómeno de la road movie española, aunque como indica su título no se ciñe en exclusiva a las películas, y estudia el road genre también en la literatura. 
Notas

1 Entre las excepciones, autores que prescinden de una acotación histórica y/o geográfica, podemos citar a Devin Orgeron, que considera la road movie un fenómeno sin fronteras unido al desarrollo de la movilidad: «More than a study of any single generic category, its attending history, or its iconography, Road Movies makes a case for the cinema's trans-national, trans-historical and trans-generic attraction to the subject of transportation» (Orgeron, 2008: 2).

2 Con un título verdaderamente emblemático: Easy Rider (Easy Rider. En busca de mi destino, Dennis Hopper, 1969).

3 Citan películas como It Happened One Night (Sucedió una noche, Frank Capra, 1934) y The Grapes of Wrath (Las uvas de la ira, John Ford, 1940).

4 Dos rasgos esenciales tomados del cine europeo configuran la estética New Hollywood: la incorporación de nuevas técnicas y procedimientos como el predominio del rodaje en escenarios naturales con equipos menos pesados, cámara al hombro, camera car, etc. (que favorecen el recurso al plano-secuencia); y la crisis de la coherencia narrativa clásica, basada en presentar de forma más o menos clara la evolución de un personaje (héroe) enfrentado a un conflicto que finalmente consigue superar (planteamiento-nudo-desenlace), en favor del interés por mostrar ambientes y fenómenos característicos de la época desde la óptica del documental. No en vano numerosas películas de los «nuevos cines» se articulan en torno al viaje por carretera, reflexionando de una u otra forma sobre la identidad nacional, rasgo por otro lado característico de toda la modernidad cinematográfica europea desde el neorrealismo italiano: Smultronstället (Fresas salvajes, Ingmar Bergman, 1957), À bout de souffle (Al final de la escapada, Jean-Luc Godard 1959), Il sorpasso (La escapada, Dino Risi, 1962), Pierrot le fou (Pierrot el loco, Jean-Luc Godard, 1965) o Stress es tres tres (Carlos Saura, 1968).

5 Este autor clasifica los géneros en canónicos, híbridos e intergéneros. Los géneros canónicos son los del cine clásico: drama o melodrama, western, comedia, musical, terror, fantástico o ciencia-ficción, aventuras o acción y criminal (cine negro, policiaco, thriller). Dentro de cada género canónico caben unas especializaciones o géneros menores, y subgéneros o ciclos (comedia romántica, aventuras exóticas). Los géneros híbridos acogen aquellas películas que participan de más de uno de los géneros canónicos: comedia dramática, péplum (drama histórico de la Antigüedad + aventuras), catástrofes (drama + aventuras). Los intetergéneros comprenden catálogos de películas que pueden pertenecer indistintamente a uno o más de los géneros canónicos pero que se identifican por un tema específico (cine de mujeres) o por una estructura concreta (películas de carretera) (Sánchez Noriega, 2002: 99-101).

6 Recordemos que en EE. UU. el género surgió coincidiendo con la profunda crisis de valores provocada por acontecimientos tan graves como el asesinato de importantes líderes políticos (Martin Luther King y el senador Robert Kennedy, ambos en 1968) o el recrudecimiento de la guerra (televisada) de Vietnam (matanza de My Lai en 1968, invasión de Camboya en 1970).

7 No obstante, consideramos desafortunado denominar a estas películas road movies, a pesar de tratarse de una tendencia generalizada dentro de la bibliografía académica. Laderman (2002), al afrontar el estudio de la road movie europea, incluye tres títulos de los 50 y 60 anteriores a Easy Rider: La Strada (Federico Fellini, 1954), Smultronstället y Weekend (Jean-Luc Godard, 1967). La postura de Laderman resulta cuando menos contradictoria si tenemos en cuenta que este capítulo aparece como cierre de su libro, tras haber dedicado la práctica totalidad del volumen al origen y evolución de la road movie dentro de EE. UU. Aquí defendemos que la road movie tiene una primera formulación estadounidense (a finales de los 60) y a partir de la década siguiente se difunde a otras cinematografías.

8 Con ánimo de ser exhaustivos, a estas habría que sumarle El alijo (Ángel del Pozo, 1976), estrenada antes que El puente, thriller con «destape» en el que dos camioneros transportan a unos emigrantes clandestinos desde Portugal a Francia. En cualquier caso se trata de un título mucho menos emblemático que los anteriores y en el que la poética del modelo norteamericano aparece más desdibujada.

9 A pesar de que muchos, como Cerón (1998: 238), hayan creído conveniente ponerle esta etiqueta a El puente.

10 A la incorporación del protagonismo femenino en Thelma and Louise enseguida le siguieron todo tipo de minorías: gays en The Living End (Vivir hasta el fin, Gregg Araki, 1992), indios americanos en Smoke Signals (Señales de humo, Chris Eyre, 1998), afroamericanos en Get on the Bus (La marcha del millón de hombres, Spike Lee, 1996), enfermos en The Sunchaser (Michael Cimino, 1996), etc.

11 La película más taquillera del cine español hasta que llegó Torrente, el brazo tonto de la ley (Santiago Segura, 1998).

12 La «matrícula española» sirve para identificar las películas puestas en pie por productoras españolas, pero estas no tienen por qué participar siempre de las formas culturales españolas, como sucede en La muerte tenía un precio (Sergio Leone, 1965), la película «española» más vista de toda la historia. Pero puede darse el caso contrario: un cineasta como Buñuel, que desarrolla casi toda su trayectoria en el extranjero (solo dirigió tres películas «matriculadas en España»), participa plenamente de esas formas autóctonas (Zunzunegui, 2005a: 9-10).

13 Una cartografía que el propio Zunzunegui utiliza como matriz para organizar su estudio sobre el cine de los sesenta, reemplazando el predominio de los criterios externos (fundamentalmente los de tipo político) por los internos "que permitan articular una historia de las formas cinematográficas del cine español» (Zunzunegui, 2005b: 34).

14 De hecho, su interés inicial (Pérez, 2007 y 2008b), se centró en estudiar el impacto en la literatura de un modelo transnacional, preferentemente cinematográfico (la road movie norteamericana): «[...] Eduardo Iglesias's Aventuras de Manga Ranglán (1992), and Por las rutas los viajeros responden a las plegarias (1996), Ignacio Martínez de Pisón's Carreteras secundarias (1995), Ray Loriga's La pistola de mi hermano (initially published as Caídos del cielo) (1995), José Machado's A dos ruedas (1996), Elvira Lindo's Manolito Gafotas on the Road (1998), David Trueba's Cuatro amigos (1998), and Cristóbal Ruiz's El loco Wonder (1999) can be considered road narratives in a strict sense [...]” (Pérez, 2008b: 131). 


\section{Bilbiografía}

ARCHER, Neil (2013), The French Road Movie. Space, Mobility, Identity, Berghahn, Nueva York.

CERÓN GÓMEZ, Juan Francisco (1998), El cine de Juan Antonio Bardem, Universidad de Murcia, Murcia.

COHAN, Steven y HARK, Ina Rae (1997), «Introduction», en COHAN, Steven \& HARK, Ina Rae (eds.), The Road Movie Book, Routledge, Nueva York, pp. 1-14.

CORRIGAN, Timothy (1991), "Genre, Gender and Hysteria: The Road Movie in Outer Space», en CORRIGAN, Timothy, A Cinema Without Walls: Movies and Culture after Vietnam, Rutgers University, New Brunswick, pp. 137-60.

DUPUY, Gabriel (1995), Les territoires de l'automobile, Anthropos, Paris.

EUVRARD, Michel (1988), «\|l n'y a plus d'Eldorados ou Les avatars du «road movie»: petit supplément au «cinéma de l'errance» pour Annie Goldman», 24 images, n. ${ }^{\circ}$ 37, pp. 30-34.

EYERMAN, Ron y LÖFGREN, Orvar (1995), «Romancing the road: Road Movies and Images of Mobility», Theory, Culture and Society, vol. 12, SAGE, Londres, Thousand Oaks y Nueva Delhi, pp. 53-79.

FENDLER, Ute (2008), «Le road movie dans le contexte interculturel africain», Cinémas, vol. 18, n. ${ }^{\circ}$ 2-3, printemps, pp. 69-88.

HERRERA, Javier y MARTíNEZ-CARAZO Cristina (eds.) (2007), Hispanismo y cine, Iberoamericana/Vervuert, Madrid.

HIGBEE, Will y LIM, Song Hwee (2010), «Concepts of Transnational Cinema: towards a Critical Transnationalism in Film Studies», Transnational Cinemas, vol. 1, n. ${ }^{\circ}$, pp. 7-21.

INDURÁIN ERASO, Carmen (2005), «Riding on Divergent but Similar Roads: Airbag or the Spanish Experience of the American Road Movie», Estudios Ingleses de la Universidad Complutense, vol. 13, pp. 107-21.

LADERMAN, David (2002), Driving Visions. Exploring the Road Movie, University of Texas, Austin.

LIE, Nadia (2011), «Saura y el género "road movie”: análisis de Stress es tres, tres», en LEFERE, Robin (ed.), Carlos Saura: una trayectoria ejemplar, Visor, Madrid, pp. 29-45.

MAZIERSKA, Ewa y RASCAROLI, Laura (2006), Crossing New Europe: Postmodern Travel and The European Road Movie, Wallflower, Londres.

MITTMAN, Elizabeth (2003), «Fantasizing Integration and Escape in the Post-Unification Road Movie», en HALLE, Randall \& MCCARTHY, Margaret (eds.), Light Motives: German Popular Film in Perspective, Wayne State University Press, Detroit, pp. 326-48.

MOSER, Walter (2008), «Présentation. Le road movie: un genre issu d'une constellation moderne de locomotion et de médiamotion», Cinémas, vol. 18, n. ${ }^{\circ}$ 2-3, primavera, pp. 7-30.

NESTINGEN, Andrew (2005), «Aki Kaurismäki's Crossroads: National Cinema and the Road Movie», en NESTINGEN, Andrew \& ELKINGTON, Trevor G. (eds.), Transnational Cinema in a Global North: Nordic Cinema in Transition, Wayne State University Press, Detroit, pp. 279-306.

ORGERON, Devin (2008), Road Movies. From Muybridge and Mèlies to Lynch and Kiarostami, Palgrave Macmillan, Nueva York.

PÉREZ, Jorge (2007), «Reckless Driving: Speed, Mobility and Transgression in the Spanish Rock 'n' Road Novel», en HENSELER, Christine \& POPE, Randolph (eds.), Generation X Rocks, Vanderbilt UP, pp. 153-169.

- (2008a), «Spanish Women Behind the Wheel: Gendering the Transition to Democracy in Vámonos, Bárbara», Revista de Estudios Hispánicos, vol. 42, n. ${ }^{\circ}$ 2, pp. 215-236.

- (2008b), «The Spanish Novel on the Road: Mobile Identities at the Turn of the Century», Anales de la literatura española contemporánea, vol. 33, n. ${ }^{\circ}$, pp. 127-151

- (2011), Cultural Roundabouts: Spanish Film and Novel on the Road, Bucknell University Press, Lewisburg.

PERRIAM, Chris; SANTAOLALLA, Isabel y EVANS, Peter W. (2007), «The Transnational in Iberian and Latin American Cinemas: Editor's Introduction», Hispanic Research Journal, vol. 8, n. ${ }^{1}$, pp. 3-9.

PICKEN, Susan (1999), «Highways, by-ways and lay-bys: The Great British Road Movie», en SARGEANT, Jack y WATSON, Stephanie (eds.), Lost Highways. An Illustrated History of the Road Movie, Creation Books, London, pp. 221-30.

POHL, Burkhard (2007), «Rutas transnacionales: la road movie en el cine español», Hispanic Research Journal, vol. 8, n. ${ }^{\circ}$, pp. 53-68. 
SÁNCHEZ NORIEGA, José Luis (2002), Historia del cine. Teoría y géneros cinematográficos, fotografía y televisión, Alianza, Madrid. SORLIN, Pierre (1996), Cines europeos, sociedades europeas 1939-1990, Paidós, Barcelona.

ZUNZUNEGUI, Santos (2002), Historias de España. De qué hablamos cuando hablamos de cine español, Ediciones de la Filmoteca, Valencia.

- (2005a), Los felices sesenta. Aventuras y desventuras del cine español (1959-1971), Paidós, Barcelona.

- (2005b), «Las vetas creativas del cine español», en POYATO, Pedro (comp.), Historia(s), motivos y formas del cine español, Plurabelle, Córdoba.

- (2011), «Prólogo», en PÉREZ RUBIO, Pablo y HERNÁNDEZ RUIZ, Javier, Escritos sobre cine español. Tradición y géneros populares, Institución «Fernando el Católico», Zaragoza. 